PROCEEDINGS OF THE

AMERICAN MATHEMATICAL SOCIETY

Volume 138, Number 6, June 2010, Pages 1941-1947

S 0002-9939(10)10227-5

Article electronically published on February 9, 2010

\title{
FILTERED ALGEBRAIC ALGEBRAS
}

\author{
ALON REGEV
}

(Communicated by Birge Huisgen-Zimmermann)

\begin{abstract}
Small and Zelmanov posed the question whether every element of a graded algebra over an uncountable field must be nilpotent, provided that the homogeneous elements are nilpotent. This question has recently been answered in the negative by Smoktunowicz. In this paper we prove that the answer is affirmative for associated graded algebras of filtered algebraic algebras. Our result is based on Amitsur's theorems on algebras over infinite fields.
\end{abstract}

\section{INTRODUCTION}

Throughout this paper, $k$ is a field and $A$ is a $k$-algebra.

\section{Definition 1.1.}

(1) If $A$ is a $k$-algebra, we say that $A$ is nil if every element of $A$ is nilpotent.

(2) If $A$ is a graded $k$-algebra, we say that $A$ is graded-nil if every homogeneous element of $A$ is nilpotent.

Clearly, every nil, graded algebra is graded-nil. The question of whether the converse to this statement is true has been of some interest.

Bartholdi [3] constructed a graded-nil but not nil algebra over a countable field. Small and Zelmanov [5] posed the question whether, for an uncountable field, every graded-nil algebra is nil. This was answered in the negative by A. Smoktunowicz 9, who constructed an algebra over any field which is graded-nil but not nil.

Let $A=\bigcup_{i \geq 0} F_{i}$ be a filtered $k$-algebra. Let gr $A$ be the associated graded algebra, and let $(\operatorname{gr} A)_{\geq 1}=\frac{F_{1}}{F_{0}} \oplus \frac{F_{2}}{F_{1}} \oplus \ldots$ be the positive part of gr $A$. Our main result is the following:

Theorem 1.2. If $k$ is uncountable and $A$ is algebraic, then $\operatorname{gr} A)_{\geq 1}$ is nil.

Thus, being graded-nil does imply being nil, for associated graded algebras of algebraic algebras over uncountable fields.

This result relates to the open question of whether there exists an affine division algebra over an uncountable field. Suppose that such an algebra exists, and suppose further that this algebra has a finite Gelfand-Kirillov dimension. By [2], it is algebraic. Then the positive part of the associated graded algebra is nil and has the same GK dimension as the original algebra. Thus the problem is reduced to the existence of a nil algebra of the same GK dimension.

Received by the editors April 24, 2009, and, in revised form, August 13, 2009, and September 14, 2009.

2010 Mathematics Subject Classification. Primary 16S15, 16 U99.

(C)2010 American Mathematical Society Reverts to public domain 28 years from publication 
The proof of Theorem 1.2 is based on Amitsur's Theorem 1.4 below.

\section{Definition 1.3.}

(1) If $A$ is a nil $k$-algebra, we say that $A$ is locally of bounded index (LBI) over $k$ if the elements of every finite-dimensional $k$-subspace of $A$ have bounded index of nilpotence.

(2) If $A$ is an algebraic $k$-algebra, we say that $A$ is locally of bounded degree $(L B D)$ over $k$ if the elements of every finite-dimensional $k$-subspace of $A$ have bounded degree of algebraicity.

In [1] Amitsur proved

Theorem 1.4 (11, Theorem 5]). Let $k$ be an uncountable field and let $A$ be a $k$ algebra. If $A$ is nil, then it is $L B I$ over $k$. If $A$ is algebraic over $k$, then it is $L B D$ over $k$.

This paper is part of the author's Ph.D. dissertation at the University of California, San Diego, written under the supervision of Lance Small, to whom he wishes to express his warm thanks.

\section{The POLYNOMIALS $p_{i_{1}, \ldots, i_{m}}$}

Throughout this paper $k$ is a field and $k\left\langle x_{1}, \ldots, x_{m}\right\rangle$ is the associative algebra of the noncommutative polynomials in $x_{1}, \ldots, x_{m}$. Note that if $A=k\left\langle x_{1}, \ldots, x_{m}\right\rangle$, then

$$
A=\bigoplus_{n=0}^{\infty} A_{n}
$$

where $A_{n}$ are the homogeneous polynomials in $x_{1}, \ldots, x_{m}$ of total degree $n$.

The order-symmetric polynomials $p_{i_{1}, \ldots, i_{m}}\left(x_{1}, \ldots, x_{m}\right)$ are defined as follows, for any $i_{1}, \ldots, i_{k} \in \mathbb{N}$.

Definition 2.1. We define $p_{i_{1}, \ldots, i_{m}}\left(x_{1}, \ldots, x_{m}\right)$ to be the sum of all the $\frac{\left(i_{1}+\ldots+i_{m}\right) !}{i_{1} ! \cdots i_{m} !}$ different monomials consisting of exactly $i_{j}$ occurrences of $x_{j}$ for each $j$. We take $p_{0, \ldots, 0}\left(x_{1}, \ldots, x_{m}\right)=1$.

Definition 2.2. For any $n \geq 0$, any field $k$ and any $x_{1}, \ldots, x_{m}$, write

$$
P_{n}\left(x_{1}, \ldots, x_{m}\right)=\operatorname{span}_{k}\left\{p_{i_{1}, \ldots, i_{m}}\left(x_{1}, \ldots, x_{m}\right) \mid i_{1}+\ldots+i_{m}=n\right\} .
$$

The polynomials $p_{i_{1}, \ldots, i_{m}}$ can be used as follows. It is well known that for $f=\alpha_{1} x_{1}+\ldots+\alpha_{m} x_{m}$,

$$
f^{n}=\sum_{1 \leq j_{1}, \ldots, j_{n} \leq m}\left(\alpha_{j_{1}} x_{j_{1}}\right) \cdots\left(\alpha_{j_{n}} x_{j_{n}}\right)=\sum_{1 \leq j_{1}, \ldots, j_{n} \leq m} \alpha_{j_{1}} \cdots \alpha_{j_{n}} \cdot x_{j_{1}} \cdots x_{j_{n}} .
$$

Lemma 2.3. Let $\alpha_{1}, \ldots, \alpha_{m} \in k$ and let $f=\alpha_{1} x_{1}+\ldots+\alpha_{m} x_{m}$. Then

$$
f^{n}=\sum_{i_{1}+\ldots+i_{m}=n} \alpha_{1}^{i_{1}} \cdots \alpha_{m}^{i_{m}} \cdot p_{i_{1}, \ldots, i_{m}}\left(x_{1}, \ldots, x_{m}\right) .
$$

Proof. This follows from (2.3) and Definition 2.1, since for each monomial $x_{j_{1}} \cdots x_{j_{n}}$ that appears in $p_{i_{1}, \ldots, i_{m}}$, the coefficient $\alpha_{j_{1}} \cdots \alpha_{j_{n}}$ in (2.3) is equal to $\alpha_{1}^{i_{1}} \cdots \alpha_{m}^{i_{m}}$.

Proposition 2.4. Let $W=\operatorname{span}_{k}\left\{\left(\alpha_{1} x_{1}+\ldots+\alpha_{m} x_{m}\right)^{n} \mid \alpha_{1}, \ldots, \alpha_{m} \in k\right\}$. If $|k| \geq n+1$, then $W=P_{n}\left(x_{1}, \ldots, x_{m}\right)$. 
Proof. By Lemma 2.3. $W \subseteq P_{n}\left(x_{1}, \ldots, x_{m}\right)$. The converse follows from [7, Lemma 1].

Corollary 2.5. Let $A$ be a $k$-algebra with $|k| \geq n+1$ and let $a_{1}, \ldots, a_{m} \in A$. Let $W \subseteq A$ be a subspace of $A$. If

$$
\left(\alpha_{1} a_{1}+\ldots+\alpha_{m} a_{m}\right)^{n} \in W
$$

for all $\alpha_{1}, \ldots, \alpha_{m} \in k$, then $P_{n}\left(a_{1}, \ldots, a_{m}\right) \subseteq W$.

Proof. Let $W_{x}=W\left(x_{1}, \ldots, x_{m}\right)=\operatorname{span}_{k}\left\{\left(\alpha_{1} x_{1}+\ldots+\alpha_{m} x_{m}\right)^{n} \mid \alpha_{1}, \ldots, \alpha_{m} \in k\right\}$ and similarly $W_{a}=W\left(a_{1}, \ldots, a_{m}\right)=\operatorname{span}_{k}\left\{\left(\alpha_{1} a_{1}+\ldots+\alpha_{m} a_{m}\right)^{n} \mid \alpha_{1}, \ldots, \alpha_{m} \in\right.$ $k\}$, so that by assumption $W_{a} \subseteq W$. Since $|k| \geq n+1$, by Proposition $2.4 W_{x}=$ $P_{n}\left(x_{1}, \ldots, x_{m}\right)$. Replacing $x_{r}$ by $a_{r}$ implies that $W_{a}=P_{n}\left(a_{1}, \ldots, a_{m}\right)$, which completes the proof.

Proposition 2.6. Let $A$ be an algebra over an infinite field $k$, and let $a_{1}, \ldots, a_{m} \in$ $A$. Then the subspace $U=k a_{1}+\ldots+k a_{m} \subseteq A$ is nil of bounded index at most $n$ if and only if $P_{n}\left(a_{1}, \ldots, a_{m}\right)=\{0\}$.

Proof. By definition $U=k a_{1}+\ldots+k a_{m}$ is nil of bounded index at most $n$ if and only if for all $\alpha_{1}, \ldots, \alpha_{m} \in k,\left(\alpha_{1} a_{1}+\ldots+\alpha_{m} a_{m}\right)^{n}=0$. Thus the "if" part of the proposition follows from Lemma 2.3, and the converse is a direct consequence of Corollary 2.5 with $W=\{0\}$.

\section{Algebraicity of Bounded Degree}

Recall the space $P_{n}\left(x_{1}, \ldots, x_{m}\right)$ of Definition 2.2 .

Definition 3.1. We write

$$
P_{\leq r}\left(x_{1}, \ldots, x_{m}\right)=\sum_{n=1}^{r} P_{n}\left(x_{1}, \ldots, x_{m}\right)=\bigoplus_{n=1}^{r} P_{n}\left(x_{1}, \ldots, x_{m}\right)
$$

and

$$
P_{\geq r}\left(x_{1}, \ldots, x_{m}\right)=\sum_{n=r}^{\infty} P_{n}\left(x_{1}, \ldots, x_{m}\right)=\bigoplus_{n=r}^{\infty} P_{n}\left(x_{1}, \ldots, x_{m}\right) .
$$

Similarly, for a $k$-algebra $A$ and elements $a_{1}, \ldots, a_{m} \in A$, let $P_{\leq r}\left(a_{1}, \ldots, a_{m}\right)=$ $\sum_{n=1}^{r} P_{n}\left(a_{1}, \ldots, a_{m}\right)$ and $P_{\geq r}\left(a_{1}, \ldots, a_{m}\right)=\sum_{n=r}^{\infty} P_{n}\left(a_{1}, \ldots, a_{m}\right)$.

Lemma 3.2. Let $D \geq d$. Let $A$ be a $k$-algebra with $|k| \geq D+1$ and let $a_{1}, \ldots, a_{m} \in$ A. Assume that for all $\alpha_{1}, \ldots, \alpha_{m} \in k, \alpha_{1} a_{1}+\ldots+\alpha_{m} a_{m}$ is algebraic of degree at most d. Then $P_{D}\left(a_{1}, \ldots, a_{m}\right) \subseteq P_{\leq d-1}\left(a_{1}, \ldots, a_{m}\right)$.

Proof. Write $P_{j}=P_{j}\left(a_{1}, \ldots, a_{m}\right)$ for any $j$, and define $P_{\geq j}$ similarly. Let $u=$ $\alpha_{1} a_{1}+\ldots+\alpha_{m} a_{m}$. By algebraicity $u^{d}$ is a linear combination of lower powers of $u$, therefore $u^{d} \in P_{\leq d-1}$. By Corollary 2.5 with $W=P_{\leq d-1}$, it follows that $P_{d} \subseteq P_{\leq d-1}$.

If $D+1 \geq d+2$, we can continue: By the same argument $P_{d+1} \subseteq P_{\leq d}$, while the previous step implies that $P_{\leq d} \subseteq P_{\leq d-1}$; hence $P_{d+1} \subseteq P_{\leq d-1}$. Continuing in this way we finally obtain $P_{D} \subseteq P_{\leq d-1}$.

Corollary 3.3. Let $k$ be infinite, let $A$ be a $k$-algebra and let $a_{1}, \ldots, a_{m} \in A$. If each element of $k a_{1}+\ldots+k a_{m}$ is algebraic of degree at most d, then $P_{\geq 0}\left(a_{1}, \ldots, a_{m}\right) \subseteq$ $P_{\leq d-1}\left(a_{1}, \ldots, a_{m}\right)$ and, in particular, $\operatorname{dim}_{k} P_{\geq 0}\left(a_{1}, \ldots, a_{m}\right)<\infty$. 
Definition 3.4. Let

$$
M_{d, m}=\left(\begin{array}{c}
d+m-1 \\
m
\end{array}\right)
$$

We note that

$$
\operatorname{dim}_{k} P_{n}\left(x_{1}, \ldots, x_{m}\right)=\left(\begin{array}{c}
m+n-1 \\
m-1
\end{array}\right)
$$

and

$$
\operatorname{dim}_{k} P_{\leq n}\left(x_{1}, \ldots, x_{m}\right)=\sum_{j=0}^{n} \operatorname{dim}_{k} P_{j}=\sum_{j=0}^{n}\left(\begin{array}{c}
j+m-1 \\
m-1
\end{array}\right)=\left(\begin{array}{c}
n+m \\
m
\end{array}\right) .
$$

Thus, given an algebra $A$ and elements $a_{1}, \ldots, a_{m} \in A$, we have

$$
\operatorname{dim}_{k} P_{\leq d-1}\left(a_{1}, \ldots, a_{m}\right) \leq M_{d, m} .
$$

Lemma 3.5. If $P_{\leq M_{d, m}}\left(a_{1}, \ldots, a_{m}\right) \subseteq P_{\leq d-1}\left(a_{1}, \ldots, a_{m}\right)$ for some $d \geq 1$, then the subspace $k a_{1}+\ldots+k a_{m}$ is algebraic of degree at most $M_{d, m}$.

Proof. Let $a=\alpha_{1} a_{1}+\ldots+\alpha_{m} a_{m} \in k a_{1}+\ldots+k a_{m}$. Then by assumption, for any $0 \leq n \leq M_{d, m}$ we have (using Lemma 2.3 with $x_{i}$ replaced by $a_{i}$ )

$$
a^{n} \in P_{n}\left(a_{1}, \ldots, a_{m}\right) \subseteq P_{\leq M_{d, m}}\left(a_{1}, \ldots, a_{m}\right) \subseteq P_{\leq d-1}\left(a_{1}, \ldots, a_{m}\right) .
$$

Thus

$$
\operatorname{dim}_{k} \operatorname{span}_{k}\left\{a^{n} \mid 0 \leq n \leq M_{d, m}\right\} \leq \operatorname{dim}_{k} P_{\leq d-1}\left(a_{1}, \ldots, a_{m}\right) \leq M_{d, m} .
$$

Therefore the set $\left\{a^{n} \mid 0 \leq n \leq M_{d, m}\right\}$ is linearly dependent over $k$, and hence $a$ is algebraic of degree at most $M_{d, m}$.

The following corollaries are immediate.

Corollary 3.6. If $P_{\geq 0}\left(a_{1}, \ldots, a_{m}\right) \subseteq P_{\leq d-1}\left(a_{1}, \ldots, a_{m}\right)$ for some $d \geq 1$, then $k a_{1}+\ldots+k a_{m}$ is algebraic of degree at most $M_{d, m}$.

Corollary 3.7. If $\operatorname{dim}_{k} P_{\geq 0}\left(a_{1}, \ldots, a_{m}\right)<\infty$, then $k a_{1}+\ldots+k a_{m}$ is algebraic of bounded degree.

We can now prove

Proposition 3.8 (4, Lemma 16(i)]). Let $A$ be an algebra over an infinite field $k$, and let $a_{1}, \ldots, a_{m} \in A$. Then the subspace $k a_{1}+\ldots+k a_{m}$ is algebraic of bounded degree if and only if $\operatorname{dim}_{k} P_{\geq 0}\left(a_{1}, \ldots, a_{m}\right)<\infty$.

Proof. One direction of the proof is given by Corollary 3.3, and the opposite direction by Corollary 3.7

\section{Filtered Algebraic algebras}

Let $A$ be a filtered $k$-algebra with the filtration $\left\{F_{n}\right\}_{n>0}$. We aim to prove that if $k$ is uncountable and $A$ is algebraic, then every element of $(\operatorname{gr} A)_{\geq 1}$ is nilpotent. In particular, being graded-nil does imply being nil, for associated graded algebras of algebraic algebras over uncountable fields.

We first note that if $a_{p_{1}}, \ldots, a_{p_{n}}$ satisfy $a_{p_{i}} \in F_{p_{i}}$ for all $1 \leq i \leq n$, then

$$
\left(a_{p_{1}}+F_{p_{1}-1}\right)\left(a_{p_{2}}+F_{p_{2}-1}\right) \cdots\left(a_{p_{n}}+F_{p_{n}-1}\right)=a_{p_{1}} a_{p_{2}} \cdots a_{p_{n}}+F_{p_{1}+\ldots+p_{n}-1} .
$$


We note the following properties of the order-symmetric polynomials in graded and filtered algebras. If the $k$-algebra $A$ is filtered by $\left\{F_{n}\right\}_{n \geq 0}$ and $a_{p}, \ldots, a_{q} \in A$ satisfy $a_{i} \in F_{i}$ for all $p \leq i \leq q$, then

$$
p_{i_{1}, \ldots, i_{m}}\left(a_{p}, \ldots, a_{q}\right) \in F_{i_{1} p+\ldots+i_{m} q},
$$

where $m=q-p+1$. Similarly, suppose $B=\bigoplus_{i>0} B_{i}$ is a graded $k$-algebra. If $b_{p}, \ldots, b_{q}$ are $m$ homogeneous elements with $b_{i} \in B_{i}^{-}$, then

$$
p_{i_{1}, \ldots, i_{m}}\left(b_{p}, \ldots, b_{q}\right) \in B_{i_{1} p+\ldots+i_{m} q} .
$$

Now let $A$ be a $k$-algebra filtered by $\left\{F_{n}\right\}_{n \geq 0}$ and let $B=\operatorname{gr}(A)$ be its associated graded algebra. Let $a_{p}, \ldots, a_{q} \in A$ satisfy $a_{i} \in F_{i}$ for each $p \leq i \leq q$, and let $b_{i}=a_{i}+F_{i-1} \in B$. We first note that by (4.1), if $p \leq p_{j} \leq q$ for all $1 \leq j \leq n$ then

$$
b_{p_{1}} \cdots b_{p_{n}}=a_{p_{1}} \cdots a_{p_{n}}+F_{p_{1}+\ldots+p_{n}-1} .
$$

Therefore if this product contains exactly $i_{j}$ occurrences of $b_{j}$ for each $p \leq j \leq q$, then

$$
b_{p_{1}} \cdots b_{p_{n}}=a_{p_{1}} \cdots a_{p_{n}}+F_{i_{p} p+\ldots+i_{q} q-1} .
$$

Since each $p_{i_{p}, \ldots, i_{q}}\left(b_{p}, \ldots, b_{q}\right)$ is a sum of such products, we have

$$
p_{i_{p}, \ldots, i_{q}}\left(b_{p}, \ldots, b_{q}\right)=p_{i_{p}, \ldots, i_{q}}\left(a_{p}, \ldots, a_{q}\right)+F_{p i_{p}+\ldots+q i_{q}-1} .
$$

Theorem 4.1. Let $A$ be a filtered $k$-algebra, with a filtration $\left\{F_{n}\right\}_{n \geq 0}$. Let $B=$ $\operatorname{gr}(A)$ be its associated graded algebra. If $A$ is $L B D$, then $B_{\geq 1}$ is LBI. In particular, if $k$ is uncountable and $A$ is a filtered algebraic $k$-algebra, then $\operatorname{gr}(A)_{\geq 1}$ is nil (and $L B I)$.

Proof. Let $S$ be a finite-dimensional subspace of $B$. We may assume $S=B_{p} \oplus \ldots \oplus$ $B_{q}$, with $1 \leq p \leq q$, since every finite-dimensional subspace of $B_{\geq 1}$ is contained in a subspace of this form. Let $b=b_{p}+\ldots+b_{q}$ be any element of $S$, where $b_{i} \in \frac{F_{i}}{F_{i-1}}$ (we take $F_{-1}=\{0\}$ ); that is, for each $i \in\{p, \ldots, q\}$, we have $b_{i}=a_{i}+F_{i-1}$, with $a_{i} \in F_{i}$. By assumption, the subspace $F_{p}+\ldots+F_{q}$ is algebraic of bounded degree, say $d$. Therefore by Corollary 3.3 , we have

$$
P_{\geq 0}\left(a_{p}, \ldots, a_{q}\right) \subseteq P_{\leq d-1}\left(a_{p}, \ldots, a_{q}\right) .
$$

Now $P_{\leq d-1}\left(a_{p}, \ldots, a_{q}\right)$ is spanned by elements of the form $p_{i_{1}, \ldots, i_{m}}\left(a_{p}, \ldots, a_{q}\right)$, with $i_{1}+\ldots+i_{m}=d-1$. Such $i_{1}, \ldots, i_{m}$ satisfy $i_{1} p+\ldots+i_{m} q \leq q(d-1)$. Therefore by (4.2) this implies

$$
P_{\geq 0}\left(a_{p}, \ldots, a_{q}\right) \subseteq F_{(d-1) q}
$$

Now let

$$
N=\left\lceil\frac{(d-1) q}{p}+1\right\rceil
$$

and suppose $i_{p}+\ldots+i_{q} \geq N$. Then

$$
p i_{p}+\ldots+q i_{q}-1 \geq p N-1 \geq(d-1) q .
$$

Therefore

$$
p_{i_{p}, \ldots, i_{q}}\left(a_{p}, \ldots, a_{q}\right) \in F_{(d-1) q} \subseteq F_{p i_{p}+\ldots+q i_{q}-1},
$$

$$
p_{i_{p}, \ldots, i_{q}}\left(b_{p}, \ldots, b_{q}\right)=p_{i_{p}, \ldots, i_{q}}\left(a_{p}, \ldots, a_{q}\right)+F_{p i_{p}+\ldots+q i_{q}-1}=0_{B}
$$


Thus $P_{N}\left(b_{1}, \ldots, b_{n}\right)=\left\{0_{B}\right\}$, and it follows from Proposition 2.6 that $\left(b_{p}+\ldots+\right.$ $\left.b_{q}\right)^{N}=0$. This shows that $S$ is nil of bounded index at most $N$. Thus $B$ is LBI. The final statement follows from Theorem 1.4.

Remark 4.2. We can use the bound $N$ of (4.9) to derive a relation between the degrees of algebraicity in $A$ and the indices of nilpotence in $\operatorname{gr}(A)$ : namely, if each

$F_{i}$ is algebraic of bounded degree at most $d_{i}$, then each $\frac{F_{1}}{F_{0}} \oplus \cdots \oplus \frac{F_{r}}{F_{r-1}}$ is nil of bounded index at most $\left(d_{r}-1\right) r+1 \leq r d_{r}$.

\section{INTEGRALity}

Definition 5.1. For a $k$-algebra $A$, let $A[x]$ be the algebra of polynomials over $x$ with coefficients in $A$. We say that an element $a(x) \in A[x]$ is integral of degree $n$ over $k[x]$ if $a(x)^{n}+p_{n-1}(x) a(x)^{n-1}+\ldots+p_{0}(x)=0$ for some $n \geq 1$ and polynomials $p_{i}(x) \in k[x]$.

Note that if $a(x)$ is integral over $k[x]$, then in particular it is algebraic over $k(x)$, the field of rational functions over $k$.

Lemma 5.2. Let $A$ be a filtered k-algebra, with filtration $\left\{A_{n}\right\}_{n \geq 0}$. Let $R=$ $\bigoplus_{n \geq 0} x^{n} A_{n} \subset A[x]$ be the Rees algebra. Let $a(x)=a_{1} x+\ldots+a_{m} x^{m} \in R$, where $a_{i} \in A_{i}$, and suppose that $a(x)$ is integral over $k[x]$. Then $a(x)^{N} \in x R$ for some $N \geq 0$.

Proof. Suppose $a(x)$ is integral over $k[x]$ of degree $n$. Then for any $N \geq 0$ we have

$$
a(x)^{N}=q_{n-1}(x) a(x)^{n-1}+\ldots+q_{1}(x) a(x)+q_{0}(x)
$$

for some polynomials $q_{i}(x) \in k[x]$. Choose $N \geq m(n-1)+1$. The left-hand side of (5.1) is in $x^{N} A[x]$. The right-hand side is in $A_{m(n-1)}[x]$ (i.e., it is a polynomial in $x$ with coefficients in the subspace $\left.A_{m(n-1)}\right)$. Thus $a(x)^{N} \in x^{N} A[x] \cap A_{m(n-1)}[x]$ $\subseteq x^{N} A_{m(n-1)}[x] \subseteq x^{N} A_{N-1}[x] \subseteq \bigoplus_{n \geq 1} x^{n} A_{n-1}=x R$.

Corollary 5.3. Let $A$ be a filtered algebra and suppose that every element of the Rees algebra $R=\bigoplus_{n \geq 0} x^{n} A_{n}$ is integral over $k[x]$. Then $\operatorname{gr}(A)_{\geq 1}$ is nil.

Proof. The map $\phi: \operatorname{gr}(A) \rightarrow \frac{R}{x R}$ defined by $\phi\left(a_{0}+\left(a_{1}+A_{0}\right)+\ldots+\left(a_{m}+A_{m-1}\right)\right)=$ $a_{0}+a_{1} x+\ldots+a_{m} x^{m}+x R$ is a graded algebra isomorphism. Under this map, an element $\left(a_{1}+A_{0}\right)+\ldots+\left(a_{m}+A_{m-1}\right) \in \operatorname{gr}(A)_{\geq 1}\left(a_{i} \in A_{i}\right)$ corresponds to $a_{1} x+\ldots+a_{m} x^{m}+x R \in \frac{R}{x R}$. By Lemma 5.2 all such elements are nilpotent.

Remark 5.4. If $A$ is a filtered $k$-algebra which satisfies the LBD property (for example, if $k$ is uncountable and $A$ is algebraic over $k$ ), then by [1, Lemma 6], the extension algebra $A \otimes_{k} k(x)$ is LBD over $k(x)$.

This raises the following question.

Question 5.5. If $A \otimes_{k} k(x)$ is LBD over $k(x)$, is every element of $A[x]$ integral over $k[x]$ ?

In view of Corollary 5.3 (and since $R \subset A[x]$ ), a positive answer would give another proof of Theorem 4.1 . 


\section{REFERENCES}

[1] S.A. Amitsur. Algebras over infinite fields. Proceedings of the American Mathematical Society, 7(1):35-48, 1956. MR0075933 (17:822b)

[2] S.A. Amitsur. Countably generated division algebras over nondenumerable fields. Bulletin of the Research Council of Israel, Section F, 7(1):39, 1957.

[3] L. Bartholdi. Branch rings, thinned rings, tree enveloping rings. Israel J. Math., 154:93-139, 2006. MR2254535 (2007k:20051)

[4] G. Bergman. Radicals, tensor products and algebraicity. Israel Mathematical Conference Proceedings, 1:150-192, 1989. MR1029309 (91d:16033)

[5] L. Small and E. Zelmanov. Private communication, 2006.

[6] A. Regev. Remarks on a theorem of Amitsur. Communications in Algebra (to appear).

[7] I. Kaplansky. On a problem of Kurosch and Jacobson. Bull. Amer. Math. Soc., 52(12):10331035, 1946. MR0019600(8:435a)

[8] I. Schur. Uber eine Klasse von Matrizen, die sich einergegebenen Matrix zuordenenlassen. Dissertation (1901); reprinted in Gesammelte Abhandlungen, Band, I, 1-72, Springer Verlag, Heidelberg, 1973. MR0462891 (57:2858a)

[9] A. Smoktunowicz. The Jacobson radical of rings with nilpotent homogeneous elements. Bull. London Math. Soc., 40:917-928, 2008. MR2471940

Department of Mathematical Sciences, Watson Hall 320, Northern Illinois UniverSity, DeKalb, Illinois 60115

E-mail address: regev@math.niu.edu 